\title{
BMJ Open \\ Task shifting interventions for cardiovascular risk reduction in low-income and middle-income countries: a systematic review of randomised controlled trials
}

\author{
Gbenga Ogedegbe, ${ }^{1,2}$ Joyce Gyamfi, ${ }^{1}$ Jacob Plange-Rhule, ${ }^{3}$ Alisa Surkis, ${ }^{4}$ \\ Diana Margot Rosenthal, ${ }^{1}$ Collins Airhihenbuwa, ${ }^{5}$ Juliet Iwelunmor, ${ }^{6}$ \\ Richard Cooper ${ }^{7}$
}

To cite: Ogedegbe G, Gyamfi J, Plange-Rhule J, et al. Task shifting interventions for cardiovascular risk reduction in low-income and middleincome countries: a systematic review of randomised controlled trials. BMJ Open 2014;4 e005983. doi:10.1136/ bmjopen-2014-005983

- Prepublication history and additional material is available. To view please visit the journal (http://dx.doi.org/ 10.1136/bmjopen-2014005983).

Received 26 June 2014 Revised 9 September 2014 Accepted 22 September 2014

\section{CrossMark}

For numbered affiliations see end of article.

Correspondence to Dr Gbenga Ogedegbe; olugbenga.ogedegbe@ nyumc.org

\section{ABSTRACT}

Objective: To evaluate evidence from published randomised controlled trials (RCTs) for the use of taskshifting strategies for cardiovascular disease (CVD) risk reduction in low-income and middle-income countries (LMICs).

Design: Systematic review of RCTs that utilised a task-shifting strategy in the management of CVD in LMICs.

Data Sources: We searched the following databases for relevant RCTs: PubMed from the 1940s, EMBASE from 1974, Global Health from 1910, Ovid Health Star from 1966, Web of Knowledge from 1900, Scopus from 1823, CINAHL from 1937 and RCTs from ClinicalTrials.gov.

Eligibility criteria for selecting studies: We focused on RCTs published in English, but without publication year. We included RCTs in which the intervention used task shifting (non-physician healthcare workers involved in prescribing of medications, treatment and/or medical testing) and nonphysician healthcare providers in the management of $\mathrm{CV}$ risk factors and diseases (hypertension, diabetes, hyperlipidaemia, stroke, coronary artery disease or heart failure), as well as RCTs that were conducted in LMICs. We excluded studies that are not RCTs.

Results: Of the 2771 articles identified, only three met the predefined criteria. All three trials were conducted in practice-based settings among patients with hypertension (2 studies) and diabetes (1 study), with one study also incorporating home visits. The duration of the studies ranged from 3 to 12 months, and the task-shifting strategies included provision of medication prescriptions by nurses, community health workers and pharmacists and telephone follow-up posthospital discharge. Both hypertension studies reported a significant mean blood pressure reduction $(2 / 1 \mathrm{~mm} \mathrm{Hg}$ and $30 / 15 \mathrm{~mm} \mathrm{Hg}$ ), and the diabetes trial reported a reduction in the glycated haemoglobin levels of $1.87 \%$. Conclusions: There is a dearth of evidence on the implementation of task-shifting strategies to reduce the

\section{Strengths and limitations of this study}

- This systematic review evaluates the effectiveness of existing task-shifting strategies in the management of cardiovascular (CV) risk factors in low-income and middle-income countries (LMICs).

- The analysis of three randomised controlled trials (the only studies meeting our eligibility criteria), where non-physician healthcare workers were involved in prescribing of medications, treatment and/or medical testing, showed significant improvement in blood pressure and glucose levels.

- The studies indicate some evidence of the effectiveness of task-shifting strategies for hypertension and diabetes management using nurses in LMICs.

- Our findings highlight the lack of data on the widespread implementation and effectiveness of task-shifting strategies for CV diseases (CVD) other than the one large trial conducted by Mendis et al at the WHO which showed that task shifting is effective at primary care healthcare facilities in Nigeria and China.

- The small number of studies and heterogeneity in terms of the various CVDs did not allow for a meta-analysis to be conducted.

burden of CVD in LMICs. Effective task-shifting interventions targeted at reducing the global CVD epidemic in LMICs are urgently needed.

\section{INTRODUCTION}

The prevalence of cardiovascular diseases (CVD) and diabetes and their attendant complications is high in low-income and middle-income countries (LMICs). According to the WHO, $80 \%$ of the mortality 
attributable to non-communicable diseases (NCDs) occurs in LMICs, and CVD account for the greatest burden. ${ }^{2}$ For example, the mortality attributable to CVD in Africa, South-East Asia and the Eastern Mediterranean regions is projected to increase from 20 to $35 \%$ by the year $2020 .^{2}$ It is estimated that more than 30 million adults in Africa have hypertension, and $75 \%$ of all deaths in Africa may be attributable to hypertension by the year $2020 .^{3}$ Stroke deaths attributable to hypertension in sub-Saharan Africa (SSA) account for a total disability of 2.6 million Disability Adjusted Life Years. ${ }^{4}$ Even more troubling is the fact that the growing NCD burden (in most LMICs) occurs in the context of high levels of infectious diseases such as malaria, HIV/ AIDS and tuberculosis, thus indicating a rapid epidemiological transition. ${ }^{5}$ This makes the urgency of addressing the epidemic of CVDs in LMICs imminent.

Although barriers to management of CVD exist at multiple levels of care, systems-level barriers (particularly the acute shortage of healthcare providers) limit the capacity of LMICs to manage CVD at the primary care level. ${ }^{1}{ }^{6-9}$ For example, although SSA has $11 \%$ of the world's population and bears over $24 \%$ of the global disease burden, it harbours only $3 \%$ of the global health workforce. ${ }^{1}$ There are 2.4 million doctors and nurses in SSA, which translates to 2 doctors and 11 nursing/midwifery personnel per 10000 people compared to 19 doctors and 49 nursing/midwifery personnel per 10000 in North America. ${ }^{10}$ Given such limited resources, costeffective approaches are urgently needed to mitigate systems-level barriers to management of CVD in LMICs. One such approach is a task-shifting strategy, defined as the rational distribution of primary care duties from physicians to non-physician healthcare providers. ${ }^{11}$ In fact, the idea of task shifting is not entirely new. Task shifting was to be the hallmark of the WHO-led primary healthcare movement of the 1980s. It was behind the declaration of what became known as health for all by the year 2000. For this purpose, and in order to maximise the efficient use of health workforce resources, primary care tasks are shifted from higher-trained health workers such as physicians to less highly trained health workers. According to the WHO and later echoed by the World Medical Association, task shifting is particularly useful in low-resource settings facing a healthcare human resource crisis, ${ }^{12}$ and is therefore proposed as a viable method for primary and secondary prevention at the primary care level. ${ }^{13}$ The benefits of task shifting are well documented in the management of HIV/AIDS. ${ }^{14}$ It utilises multiple strategies to address the CVD epidemic including screening, counselling on lifestyle modification, initiation of treatment and referral to specialist care. $^{2} 11-13$ 15-18

Despite the global call for task shifting for management of non-communicable diseases and the potential for task-shifting strategies to mitigate the systems-level barriers to implementation of primary and secondary prevention of CVD in LMICs, their effectiveness has not been widely evaluated. In this systematic review, we evaluated the evidence from published randomised controlled trials (RCTs) for the use of task-shifting strategies for CVD risk reduction in LMICs.

\section{METHODS}

\section{Search strategy}

We identified published trials that met predefined inclusion criteria using standard Cochrane Collaboration systematic review techniques ${ }^{19}$ and the Preferred Reporting Items for Systematic Reviews and Meta-Analyses (PRISMA) ${ }^{20}$ statement. We searched the following databases: PubMed from the 1940s, EMBASE from 1974 Global Health from 1910, Ovid Health Star from 1966 Web of Knowledge from 1900, Scopus from 1823 , CINAHL from 1937 and RCTs from ClinicalTrials.gov. The search strategy included terms from three subject categories: those related to CVD; those related to the concept of task shifting; and those related to LMICs, as defined by the World Bank (using a variety of factors including gross domestic product, population, economic policy and external debt, health, environment and education). ${ }^{21}$ All concepts were then combined using both keywords and controlled vocabularies such as "task shift" ${ }^{*}$ AND "balance of care OR nonphysician clinician OR substitute health worker OR community care giver OR primary health care team OR cadres OR nurs*" AND "CVD". The search terms used were similar to the ones used by Callaghan et $a l^{14}$ in their systematic review of task shifting in HIV treatment. Searches were undertaken in October 2011 and repeated in March 2013 before the final write-up.

We adopted the following definition of task shifting by Callaghan et $a l^{14}$ : "the process of shifting tasks to a variety of health workers; including nurses or new cadres in prescribing of medications and medical testing, as long as it is a streamlined, rationalized chain of care." As depicted in figure 1, the process of task shifting should involve ongoing training from higher-level health professionals, delegation and continuous supervision. Also, patients with complicated disease cases should always be referred for specialist care.

\section{Eligibility criteria for inclusion of studies}

We limited our search to only RCTs published in English, but without publication year. We included RCTs in which the intervention used task-shifting (nonphysician healthcare workers involved in prescribing of medications, treatment and/or medical testing) and non-physician healthcare providers in the management of $\mathrm{CV}$ risk factors and diseases (hypertension, diabetes, hyperlipidaemia, stroke, coronary artery disease or heart failure), as well as RCTs that were conducted in LMICs. We excluded studies that were not RCTs. We then reviewed the identified RCTs in their entirety to determine their eligibility. 


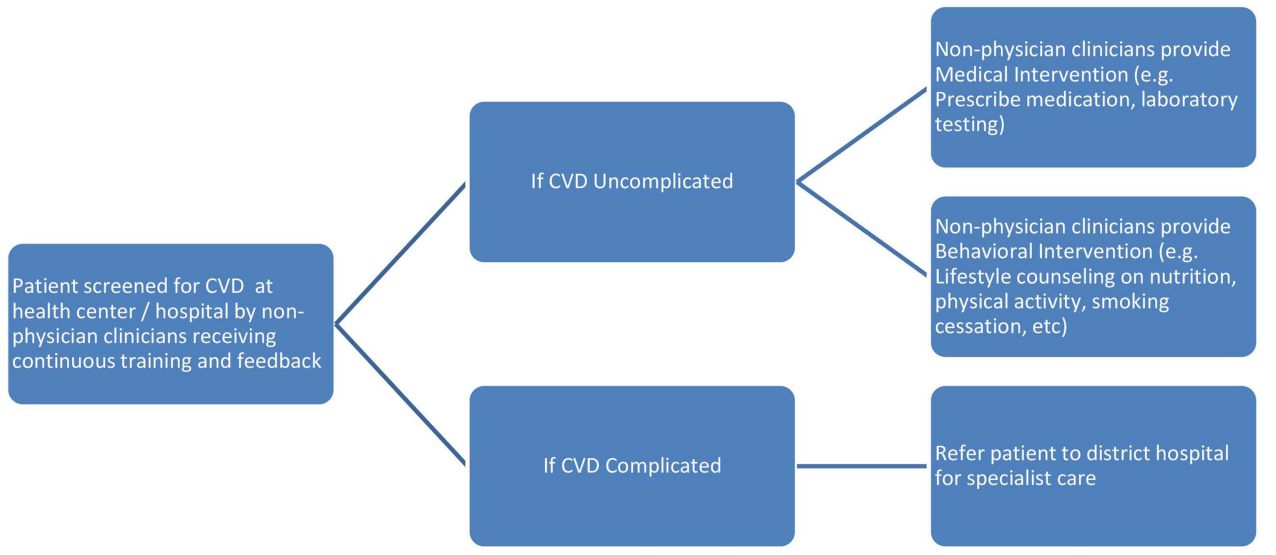

Figure 1 Referral pathway for cardiovascular diseases management using task shifting.

\section{Data extraction}

Each of the authors assessed all retrieved lists of citations and abstracts independently. Initially, we determined the relevance of the articles by title and abstract. Discrepancies between the authors about the eligibility of retrieved studies were resolved by discussion. We then obtained printed copies of all relevant articles for extensive examination to ensure that the articles met all eligibility criteria. Information from potentially eligible articles including study country, study design, methods, participant characteristics, retention rates and study outcomes were extracted into the Cochrane Review Manager. $^{22}$

\section{Quality assessment}

The quality of available RCTs was assessed using the Cochrane criteria $^{23}$ adapted from previous suggestions. ${ }^{24} 25$ Specifically, the risk of bias in generation of the randomisation sequence, allocation concealment and blinding (participants and outcome assessors), incomplete outcome data and selective reporting were assessed as adequate, uncertain or inadequate. ${ }^{24} 25$ Two authors (JG and JI) assessed the risk of bias in the individual studies that met the inclusion criteria. Disagreements were resolved by consensus, and a third reviewer was consulted if disagreements persisted.

\section{RESULTS}

Full search strategies for each of the databases are provided in online supplementary appendix 1 . We retrieved and screened 2771 articles (figure 2), and conducted full paper review on 32 articles that initially met the inclusion criteria including study location in LMICs, the use of non-physician providers to provide health services, CVD and use of task-shifting strategies. After further review, we excluded 18 articles including: studies that were not conducted in LMICs (5); studies that were missing important details about intervention strategies (4); protocol papers that were missing main trial outcomes (4); studies that referred to the same study protocol conducted in the same populations (3); studies whose primary outcome did not include major CV risk factors or CVD (1); and studies that only provided abstracts (1). A total of 14 articles met the initial eligibility criteria. ${ }^{21} 26-38$ The 14 articles were further screened based on whether or not the intervention fulfilled the definition of task shifting used for this review (use of non-physician clinicians in prescribing medications or performing medical testing in the treatment or management of CVD). This final review led to a further elimination of 11 additional articles, ${ }^{27-32}{ }^{34-38}$ leaving only three RCTs, which were included in this systematic review. ${ }^{212633}$ The characteristics of the studies included in this systematic review are presented in tables 1 and 2. One trial was conducted in Nigeria, ${ }^{26}$ other multicentre trials were conducted in Nigeria and China ${ }^{33}$ and the last one was conducted in Iran. ${ }^{21}$ The study populations were patients with hypertension (two studies) ${ }^{26} 33$ and type 2 diabetes (one study; ${ }^{21}$ table 2). The sample size of the interventions varied, with a range of 61-2397 patients (table 1): the sample size for the diabetes trial was $61^{21}$, while those of the two hypertension trials were $544^{26}$ and $23977^{33}$ The duration of these studies ranged from 3 months for the diabetes trial to $6-12$ months for the hypertension trials. The reporting quality of all three trials were rated at $73 \%$ using the Jadad quality measure. ${ }^{39}$

The overall risk of bias was moderate; the random sequence generation was adequate in $67 \%(2 / 3)$ of the studies, and allocation concealment in $67 \%(2 / 3)$; however, blinding of data collection (participants) was not possible in any of the studies due to the nature of the intervention, as well as with blinding of the outcomes except for one study. ${ }^{21}$ All the studies described the methods used to collect outcomes, although we could not assess blinding of the researchers collecting the outcome data. Overall, incomplete data were reported in all the studies; however, selective reporting was poor with very minimal information in all the studies on whether the interventions were implemented with fidelity, "that is whether each of the components of 
Reports identified from literature search $(n=2771)$

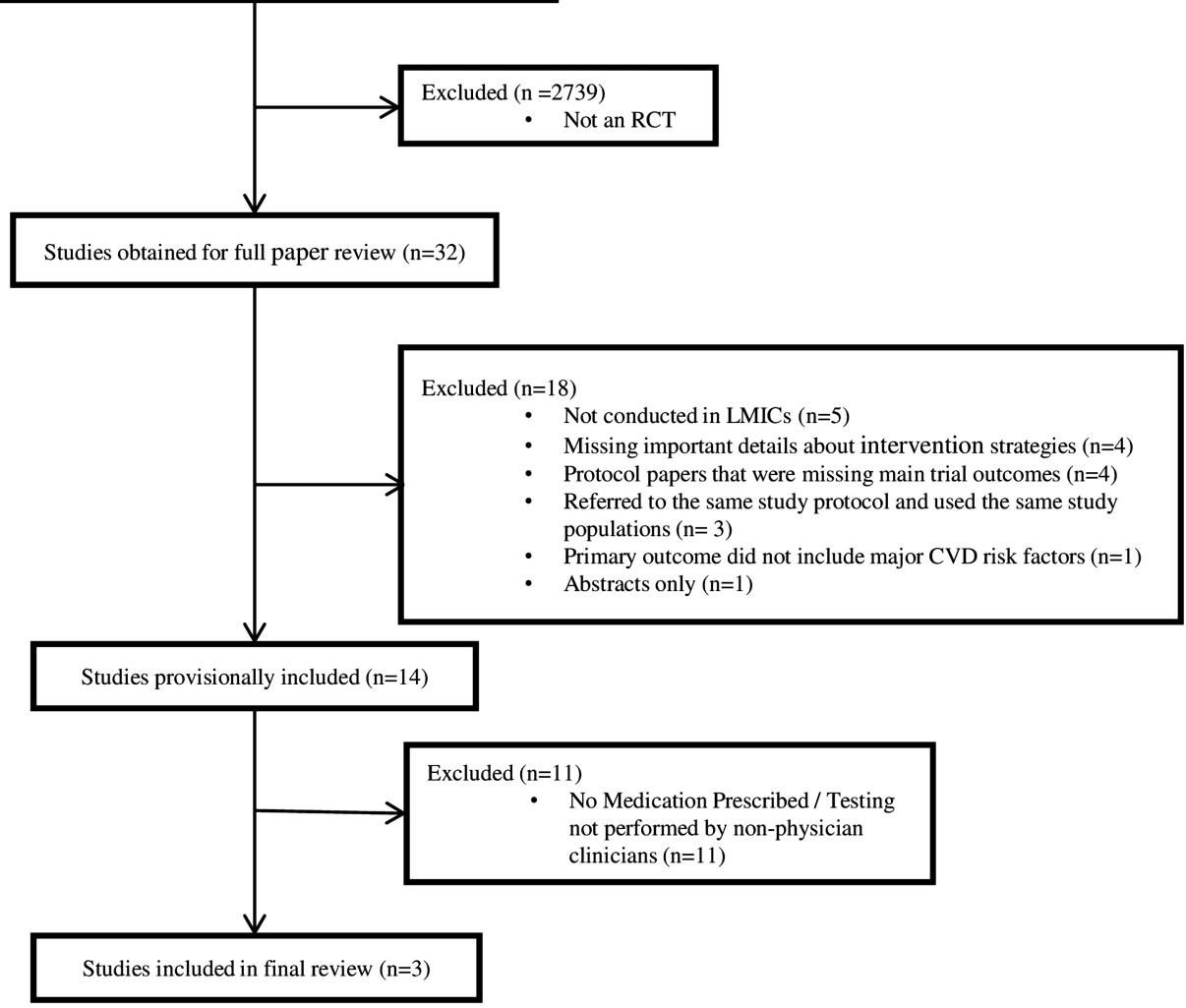

Figure 2 Flow diagram showing citations retrieved from literature searches and number of trials included in the analysis.

the intervention were delivered in a comparable manner to all participants and is true to the objectives of the underlying research." ${ }^{40}$

\section{Hypertension trials}

Both studies evaluated the effect of task shifting on hypertension control using various forms of task-shifting strategies including interventions led by nurses, pharmacists and community health workers (table 2). The studies were conducted in Nigeria and China. ${ }^{26}{ }^{33}$ In addition to nurses or pharmacists prescribing antihypertensive medications, the interventions included health education emphasising lifestyle modifications such as diet, physical activity and medication adherence. The interventions were effective at improving blood pressure (BP) control in both studies. ${ }^{2633}$

The first hypertension trial, by Adeyemo and colleagues, examined the effectiveness of the use of nurses to deliver hypertension management in a primary care practice versus usual care plus home visits on medication

Table 1 Characteristics of studies included in the systematic review

\begin{tabular}{|c|c|c|c|c|c|c|}
\hline $\begin{array}{l}\text { Study } \\
\text { (year) }\end{array}$ & $\begin{array}{l}\text { Duration of } \\
\text { interventions } \\
\text { (months) }\end{array}$ & $\begin{array}{l}\text { Sample } \\
\text { size }\end{array}$ & $\begin{array}{l}\text { Completed } \\
\text { Follow-up } \\
\text { per cent }\end{array}$ & $\begin{array}{l}\text { Completed } \\
\text { Follow-up } \\
\text { per cent }\end{array}$ & $\begin{array}{l}\text { Primary outcome } \\
\text { measures }\end{array}$ & $\begin{array}{l}\text { Statistical } \\
\text { improvement } \\
\text { in CVD }\end{array}$ \\
\hline $\begin{array}{l}\text { Adeyemo et al } \\
(2013)^{26}\end{array}$ & 6 & 544 & 88 & 72 & Medication adherence BP Control & Yes \\
\hline $\begin{array}{l}\text { Mendis et al } \\
(2010)^{33}\end{array}$ & 12 & 2397 & 93.5 & 86.4 & $\begin{array}{l}\text { Systolic BP change from Baseline } \\
\text { to } 12 \text { months }\end{array}$ & Yes \\
\hline \multirow{2}{*}{$\begin{array}{l}\text { Nesari et al } \\
(2010)^{21}\end{array}$} & 3 & 61 & 100 & 96.8 & Adherence to diabetes regimen & Yes \\
\hline & & & & & Reduction in $\mathrm{HbA}_{1 \mathrm{c}}$ levels & \\
\hline
\end{tabular}




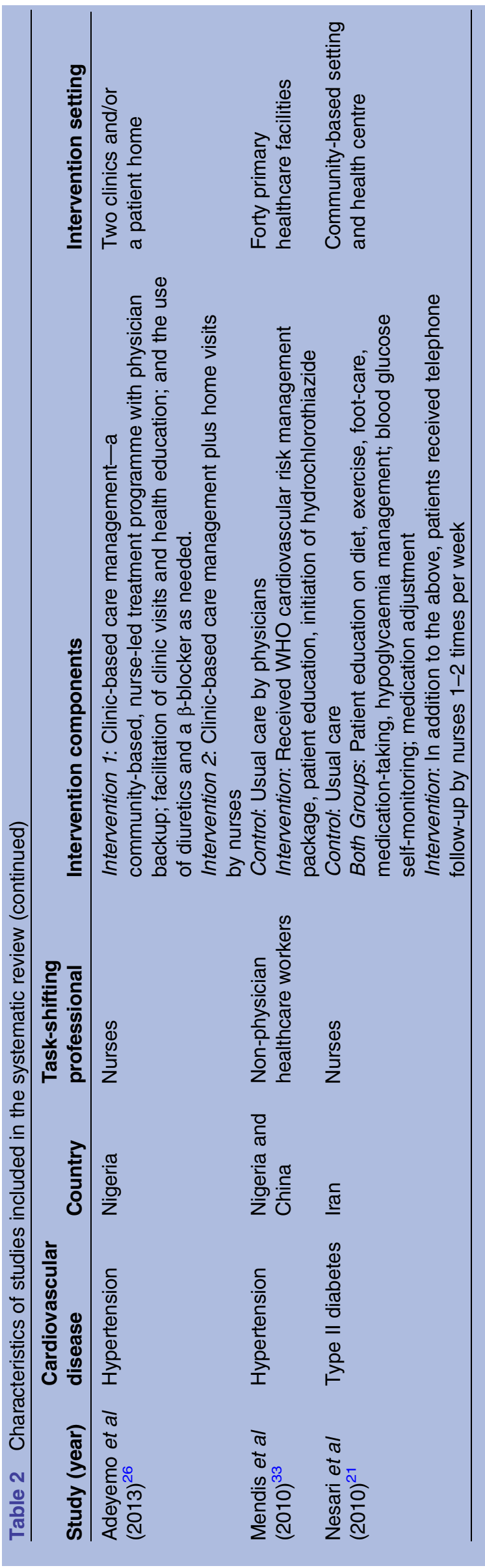

adherence, and $\mathrm{BP}$ control at 6 months among 544 patients (mean age $\sim 63$ years, mean $\mathrm{BP} \sim 168 / 92 \mathrm{~mm} \mathrm{Hg}$ ) in Nigeria. ${ }^{26}$ The intervention included the following components: (1) a nurse-led treatment programme with physician backup, (2) clinic visits and health education by nurses, (3) the use of diuretics and a $\beta$-blocker prescribed by nurses with physician backup. The primary outcome of $\mathrm{BP}$ control (BP $<140 / 90 \mathrm{~mm} \mathrm{Hg}$ ) was achieved in $66 \%$ of the study participants $(66.7 \%$ in the clinic only group, $65.4 \%$ in the clinic plus home visit; $\mathrm{p}=0.584$ and $\mathrm{p}=0.891) .{ }^{26}$ The overall decline in the mean systolic and diastolic BP over the 6-month period was $30 \mathrm{~mm} \mathrm{Hg}$ and $15 \mathrm{~mm} \mathrm{Hg}$, respectively $(\mathrm{p}<0.001$ and $\mathrm{p}<0.0001) .{ }^{26}$ Overall, medication adherence was high among study participants with $77 \%$ of participants taking $>98$ of their prescribed pills.

The second hypertension trial was the largest in this review. In this cluster RCT, Mendis $e t a l^{33}$ evaluated the effectiveness of the WHO Cardiovascular Disease (CVD) package using task-shifting strategies to improve BP control among 2397 hypertensive patients from 40 primary care facilities (20 intervention sites, 20 control sites) in Nigeria and China. Non-physician healthcare workers provided patients at the intervention sites with the WHO CVD package protocol, while those at the control sites received usual care for a period of 12 months. The WHO CVD package was designed as an adaptable, cost-effective tool for systematic case management at all healthcare levels and consequently for scaling up in health systems in LMICs. The programme provides clinical decision support for the assessment and management of $\mathrm{CV}$ risk through easy-to-follow risk-assessment algorithms, lifestyle counselling, drug treatment protocols and referral pathways. ${ }^{41}$ The protocol consists of four basic steps: inquiry about the patient's history (heart attack, angina, stroke, transient ischaemic attack, diabetes and lifestyle behaviours); physical and laboratory examination (including BP measurements, anthropometrics, urine dipstick, fasting glucose and plasma cholesterol); estimation of the patient's CVD risk based on the WHO risk charts (low, medium or high); and subsequent initiation of drug therapy and lifestyle counselling during follow-up visits. ${ }^{33}$ Depending on the patient's CVD risk, the treatment decisions include either (1) immediate referral to a specialist in the case of patents with high CVD risk; or (2) lifestyle counselling on diet, physical activity and tobacco cessation; prescription of an antihypertensive medication; and follow-up with a provider. The primary outcome was change in systolic BP from baseline to 12 months. Systolic and diastolic BP decreased significantly in favour of the intervention group at both study sites $(p<0.0001)$ and $((p<0.0002)$, but the BP control rate was abysmally low at only $20 \%$. The intervention resulted in a significantly greater reduction in systolic and diastolic BP for the treatment group (2 $\mathrm{mm} \mathrm{Hg}$ and $1 \mathrm{~mm} \mathrm{Hg}$ ) than the control group in both countries. ${ }^{33}$ 


\section{Diabetes trial}

The diabetes trial evaluated whether nurse-led care could improve diabetes management compared to usual care. ${ }^{21}$ This trial was conducted by Nesari $e t a l^{21}$ in Iran among 61 patients with diabetes, who received either telephone-based nurse follow-up care for 3 months or usual care. Both groups received health education on diet, physical activity, foot care, blood glucose selfmonitoring, management of medication side effects and hypoglycaemia. Additionally, in the intervention group, the nurse adjusted the patients' medications according to the patients' reported glycaemic level, with backup from an endocrinologist. The reported decline in glycated haemoglobin (HbAlc) was higher in the intervention group compared to the usual care group $(1.87 \%$ in the intervention group, $\mathrm{p}<0.001$; and $0.42 \%$ in the usual care group, $\mathrm{p}<0.15) .{ }^{21}$ Similarly, the mean levels of HbAlc was significantly lower in the intervention group than in the usual care group at 3 months (mean HbAlc of $7.04 \% \pm 1.18$ in the intervention group vs $8.64 \% \pm 1.88$ for the control group; $\mathrm{p}<0.001){ }^{21}$

\section{DISCUSSION}

In this review, we examined the evidence for task shifting of primary care duties for management of CVD in LMICs. We reviewed three clinical trials that utilised taskshifting strategies for management of CVD in LMICs. ${ }^{21} 2633$ Two of the three trials were hypertension studies, ${ }^{26} 33$ and one was a diabetes trial. ${ }^{21}$ The outcomes of the three trials were positive with significant improvement in BP and HbAlc. ${ }^{21} 2633$ The studies show some evidence of the effectiveness of task-shifting strategies for management of hypertension and diabetes using nurses.

Some of the common task-shifting enablers among the studies are as follows: continuous educational training and feedback from higher level health professionals; bridging hospital care to home care in order to ensure continuity of patient care; and providing explicit training tools including medication/treatment algorithms. Nonetheless, barriers to task shifting in LMICs that currently do not utilise task-shifting strategies include the lack of policy on the ability of non-physician providers to prescribe medications for common disorders; the lack of a referral system as backup for complicated cases; the lack of an organisational structure to accommodate a non-physician provider as a primary care provider; and the lack of competence of the non-physician provider in their ability to manage uncomplicated CV risk factors; and finally, the lack of infrastructure for data collection and monitoring of clinical information on a periodic basis.

The concept of task shifting is not new because taskshifting strategies have proven effective in the battle against the HIV/AIDs epidemic in LMICs ${ }^{14}$; and thus may be potentially effective for chronic disease management, provided adequate and sustainable training is afforded to the health professionals involved. Considering the barriers and challenges that task shifting may pose if non-physician healthcare workers are not equipped with the expertise to efficiently manage HIV/AIDS, the WHO launched the treat, train, retrain plan in $2006^{42}$ to ensure competency and aid in capacity building of these providers. As a result, many LMICs have adopted task-shifting strategies for HIV/AIDS management in LMICs. In this regard, Callaghan $e^{a l^{14}}$ conducted a systematic review of 84 articles on HIV treatment and care in SSA, and their findings suggest that task-shifting strategies led to improved efficiency in delivery of healthcare services, enhanced access to care, better team dynamics and improved quality of care and health outcomes for patients with HIV/AIDS.

Task shifting is a potentially viable and low-cost strategy for reducing the growing CVD epidemic in LMICs because it utilises multiple strategies that are amenable to the management of CVDs including screening, counselling on lifestyle modification, initiation of treatment and referral to specialist care. ${ }^{11-14}{ }^{41}$ We are not aware of any rigorous evaluation of task-shifting strategies for management of CVDs in LMICs. To the best of our knowledge, our study was the first systematic review to evaluate the effectiveness of existing task-shifting strategies in the management of CV risk factors in LMICs. Our findings highlight the lack of data on the widespread implementation and effectiveness of task-shifting strategies for CVD other than the one large trial conducted by Mendis $e t a l^{33}$ at the WHO, which showed that task shifting is effective at primary care healthcare facilities in Nigeria and China. The other studies reviewed had numerous weaknesses. First, the quality of the trials was low, given their very small sample sizes, poor definition of study outcomes and short duration of the trials (only 3 months for the diabetes trial ${ }^{21}$ and 6 months for one of the hypertension trials ${ }^{26}$ ), making it difficult to ascertain the effect of regression to mean on the study outcomes. Second, the authors provided a very scanty description of the non-physician healthcare providers who delivered the task-shifting duties: only two of the studies identified that nurses provided the task-shifting duties. ${ }^{21}{ }^{26}$ Unfortunately, the largest trial with the best quality did not provide any information on the level of training of the task-shifting healthcare provider. ${ }^{33}$ Third, there were no data on the cost-effectiveness of these studies, and finally, none of the trials integrated their intervention into existing healthcare systems making evaluation of the implementation and dissemination of the study findings problematic.

A possible limitation of our review is that we excluded 11 RCT studies which did not meet our criteria. These studies are shown in table 3 , and the majority of the reasons for exclusion are because they were studies that were largely patient education/health education interventions carried out by non-physicians. These duties readily fit in the nurses and other non-physician duties, and hence were not considered task shifting. Other 
Table 3 Excluded RCT studies not meeting the final review criteria

\begin{tabular}{lll}
\hline Study (year) & Country & Reason for exclusion \\
\hline Andryukhin et al $(2010)^{27}$ & Russia & Educational programme for patients with heart failure \\
DePue et al $(2013)^{28}$ & American Samoa & Diabetes management support programme \\
Hacihasanoglu et al $(2011)^{29}$ & Turkey & Hypertension health education \\
Jafar et al $(2009)^{30}$ & Pakistan & Home-based hypertension health education \\
Jafar et al $(2010)^{31}$ & Pakistan & Home-based hypertension health education \\
Jiang et al $(2007)^{32}$ & China & Cardiac rehabilitation programme \\
Selvaraj et al $(2012)^{34}$ & Malaysia & Telephone intervention for dyslipidemia patients \\
Sit et al $(2007){ }^{35}$ & China & Educational intervention for self-care management of stroke \\
Wong et al $(2010)^{36}$ & China & Health education for patients with end-stage renal disease \\
Wong et al $(2005)^{37}$ & China & Telephone intervention for patients with diabetes \\
Zhao et al $(2010)^{38}$ & China & Telephone follow-up for patients with coronary heart disease \\
\hline
\end{tabular}

reasons include the lack of randomisation (2739 studies), and although they measured outcomes of CVD, the lack of randomisation makes them low quality. These studies were nevertheless effective as pre-post design that policymakers may find useful.

Future studies should focus on the cost-effectiveness of task-shifting interventions for CVD risk reduction as part of the larger healthcare system. In addition, these studies should compare the cost-effectiveness of the use of nurses versus other allied healthcare workers. In order for task-shifting strategies to be considered effective, evidence of their implementation for addressing the CVD epidemic as part of the existing healthcare systems in LMICs is paramount. Thus, in 2001, the Global Alliance for Chronic Diseases (GACD) funded 15 implementation trials targeting hypertension control. Five of these studies evaluated the role of task-shifting strategies to reduce overall $\mathrm{CV}$ risk and improve hypertension control in Ghana, India, Kenya, Tanzania and South Africa. ${ }^{43}$ Such studies integrated into existing healthcare systems will guarantee subsequent adoption of interventions if proven successful.

In conclusion and based on our findings, task-shifting strategies are applicable and feasible in many LMICs, which are burdened with infectious and chronic diseases, compounded with limited material and healthcare personnel resources. With proper training and continuous feedback, lower level health professionals can be instrumental in managing CVDs efficiently. Future studies should address their implementation as part of existing healthcare systems as well as their costeffectiveness in LMICs.

\footnotetext{
Author affiliations

${ }^{1}$ Department of Population Health, NYU School of Medicine, NYU Langone Medical Center, New York, USA

${ }^{2}$ NYU Global Institute of Public Health, New York University, New York, USA

${ }^{3}$ School of Medical Sciences, Kwame Nkrumah University of Science and Technology, Kumasi, Ghana

${ }^{4}$ NYU Health Sciences Libraries, NYU Langone Medical Center, New York, USA

${ }^{5}$ Department of Biobehavioral Health, Pennsylvania State University, University Park, Pennsylvania, USA
}

${ }^{6}$ Department of Kinesiology and Community health, University of Illinois at Urbana-Champaign, Urbana, Illinois, USA

${ }^{7}$ Stritch School of Medicine, Loyola Chicago Medical Center, Chicago, Illinois, USA

Contributors G0 conceived of the review, participated in the design and article selections and helped to draft the manuscript. JG conducted supplementary literature review, participated in the article selections and drafted the manuscript. AS conducted the primary literature review and helped to draft the manuscript. DMR participated in the article selection process and helped to draft the manuscript. JP-R, CA, JI and RC all contributed to the article selection process and edited the manuscript for critical content. All authors have read and approved the final manuscript.

Funding This project was supported by the following grants: a U01 (U01HL114198), Dr Ogedegbe's K24 (K24 HL111315) and a Fogarty training grant (D43TW009140) from the National Institute of Health (NIH/NHLBI).

Competing interests None.

Provenance and peer review Not commissioned; externally peer reviewed.

Data sharing statement Extra data can be accessed via the Dryad data repository at http://datadryad.org/ with the doi:10.5061/dryad.565kh.

Open Access This is an Open Access article distributed in accordance with the Creative Commons Attribution Non Commercial (CC BY-NC 4.0) license, which permits others to distribute, remix, adapt, build upon this work noncommercially, and license their derivative works on different terms, provided the original work is properly cited and the use is non-commercial. See: http:// creativecommons.org/licenses/by-nc/4.0/

\section{REFERENCES}

1. Anyangwe SC, Mtonga C. Inequities in the global health workforce: the greatest impediment to health in sub-Saharan Africa. Int $J$ Environ Res Public Health 2007;4:93-100.

2. WHO. Global Status Report on Noncommunicable Diseases 2010. http://www.who.int/nmh/publications/ncd_report_full_en.pdf (accessed 15 Apr 2013).

3. Kearney PM, Whelton M, Reynolds K, et al. Global burden of hypertension: analysis of worldwide data. Lancet $2005 ; 365$ 217-23.

4. Mensah GA. Epidemiology of stroke and high blood pressure in Africa. Heart 2008;94:697-705.

5. BeLue R, Okoror TA, Iwelunmor J, et al. An overview of cardiovascular risk factor burden in sub-Saharan African countries: a socio-cultural perspective. Global Health 2009;5:10.

6. Beaglehole R, Epping-Jordan J, Patel V, et al. Improving the prevention and management of chronic disease in low-income and middle-income countries: a priority for primary health care. Lancet 2008;372:940-9.

7. Dussault G, Dubois CA. Human resources for health policies: critical component in health policies. Hum Resour Health 2003;1:1.

8. Hagopian A, Thompson MJ, Fordyce M, et al. The migration of physicians from sub-Saharan Africa to the United States of America: measures of the African brain drain. Hum Resour Health 2004;2:17. 
9. Pang $T$, Lansang MA, Haines A. Brain drain and health professionals. BMJ 2002;324:499-500.

10. Mayosi B. The 10 'Best Buys' to combat heart disease, diabetes and stroke in Africa. http://heart.bmj.com/content/early/2013/05/16/ heartjnl-2013-304130.full.pdf+htm (accessed 27 Feb 2013).

11. WMA Resolution on Task Shifting from the Medical Profession. http://www.wma.net/en/30publications/10policies/t4/ (accessed 18 Mar 2013).

12. Lekoubou A, Awah P, Fezeu L, et al. Hypertension, diabetes mellitus and task shifting in their management in sub-Saharan Africa. Int $J$ Environ Res Public Health 2010;7:353-63.

13. Zachariah R, Ford N, Philips M, et al. Task shifting in HIV/AIDS opportunities, challenges and proposed actions for sub-Saharan Africa. Trans R Soc Trop Med Hyg 2009;103:549-58.

14. Callaghan M, Ford N, Schneider H. A systematic review of taskshifting for HIV treatment and care in Africa. Hum Resour Health 2010;8:8.

15. Labhardt ND, Balo JR, Ndam M, et al. Improved retention rates with low-cost interventions in hypertension and diabetes management in a rural African environment of nurse-led care: a cluster-randomised trial. Trop Med Int Health 2011;16:1276-84.

16. Kim HS, Oh JA. Adherence to diabetes control recommendations: impact of nurse telephone calls. J Adv Nurs 2003;44:256-61.

17. Joshi R, Chow C, Raju P, et al. The Rural Andhra Pradesh Cardiovascular Prevention Study (RAPCAPS): a cluster randomized trial. J Am Coll Cardiol 2012;59:1188-96.

18. Cho JH, Kwon HS, Kim HS, et al. Effects on diabetes management of a health-care provider mediated, remote coaching system via a PDA-type glucometer and the Internet. J Telemed Telecare 2011;17:365-70.

19. Higgins JPT, Green S, eds. Cochrane Handbook for Systematic Reviews of Interventions Version 5.1.0 [updated March 2011]. The Cochrane Collaboration, 2011. www.cochrane-handbook.org

20. Moher D, Liberati A, Tetzlaff $\mathrm{J}$, et al. Preferred reporting items for systematic reviews and meta-analyses: the PRISMA statement. PLoS Med 2009;6:e1000097.

21. Nesari M, Zakerimoghadam M, Rajab A, et al. Effect of telephone follow-up on adherence to a diabetes therapeutic regimen. Jpn $J$ Nurs Sci 2010;7:121-8.

22. Review Manager (RevMan). Version 5.0. Copenhagen: The Nordic Cochrane Centre: The Cochrane Collaboration, 2008.

23. Higgins J. Green S. Cochrane handbook for systematic reviews of interventions Version 5.1. 0 [updated March 2011]. The Cochrane Collaboration, 2011. http://www cochrane-handbook org 2012.

24. Clark CE, Smith LF, Taylor RS, et al. Nurse led interventions to improve control of blood pressure in people with hypertension: systematic review and meta-analysis. BMJ 2010;341:c3995.

25. De Simoni A, Hardeman W, Mant J, et al. Trials to improve blood pressure through adherence to antihypertensives in stroke/TIA: systematic review and meta-analysis. J Am Heart Assoc 2013;2: e000251.

26. Adeyemo A, Tayo BO, Luke A, et al. The Nigerian antihypertensive adherence trial: a community-based randomized trial. $J$ Hypertens 2013;31:201-7.

27. Andryukhin A, Frolova E, Vaes B, et al. The impact of a nurse-led care programme on events and physical and psychosocial parameters in patients with heart failure with preserved ejection fraction: a randomized clinical trial in primary care in Russia. Eur $J$ Gen Pract 2010;16:205-14

28. DePue JD, Dunsiger S, Seiden AD, et al. Nurse-community health worker team improves diabetes care in American Samoa: results of a randomized controlled trial. Diabetes Care 2013;36:1947-53.

29. Hacihasanoglu R, Gozum S. The effect of patient education and home monitoring on medication compliance, hypertension management, healthy lifestyle behaviours and BMI in a primary health care setting. J Clin Nurs 2011;20:692-705.

30. Jafar TH, Hatcher J, Poulter N, et al. Community-based interventions to promote blood pressure control in a developing country: a cluster randomized trial. Ann Intern Med 2009;151:593-601.

31. Jafar TH, Islam M, Hatcher J, et al. Community based lifestyle intervention for blood pressure reduction in children and young adults in developing country: cluster randomised controlled trial. BMJ 2010;340:c2641.

32. Jiang X, Sit JW, Wong TK. A nurse-led cardiac rehabilitation programme improves health behaviours and cardiac physiological risk parameters: evidence from Chengdu, China. J Clin Nurs 2007:16:1886-97.

33. Mendis S, Johnston SC, Fan W, et al. Cardiovascular risk management and its impact on hypertension control in primary care in low-resource settings: a cluster-randomized trial. Bull World Health Organ 2010;88:412-19.

34. Selvaraj FJ, Mohamed M, Omar K, et al. The impact of a disease management program $(\mathrm{COACH})$ on the attainment of better cardiovascular risk control in dyslipidaemic patients at primary care centres (The DISSEMINATE Study): a randomised controlled trial. BMC Fam Pract 2012;13:97.

35. Sit JW, Yip VY, Ko SK, et al. A quasi-experimental study on a community-based stroke prevention programme for clients with minor stroke. J Clin Nurs 2007;16:272-81.

36. Wong FK, Chow SK, Chan TM. Evaluation of a nurse-led disease management programme for chronic kidney disease: a randomized controlled trial. Int J Nurs Stud 2010;47:268-78.

37. Wong FK, Mok MP, Chan T, et al. Nurse follow-up of patients with diabetes: randomized controlled trial. J Adv Nurs 2005;50: 391-402.

38. Zhao Y, Wong FK. Effects of a postdischarge transitional care programme for patients with coronary heart disease in China: a randomised controlled trial. J Clin Nurs 2009:18:2444-55.

39. Jadad AR, Moore RA, Carroll D, et al. Assessing the quality of reports of randomized clinical trials: is blinding necessary? Control Clin Trials 1996;17:1-12.

40. Dumas JE, Lynch AM, Laughlin JE, et al. Promoting intervention fidelity: conceptual issues, methods, and preliminary results from the EARLY ALLIANCE prevention trial. Am J Prev Med 2001:20:38-47.

41. WHO. WHO CVD risk management package for low-and med-resource settings. 2002. http://whqlibdoc.who.int/publications/ 2002/9241545852.pdf?ua=1 (accessed 15 Apr 2013)

42. WHO. Task shifting to tackle health worker shortages. http://www. who.int/healthsystems/task_shifting_booklet.pdf (accessed 20 Oct 2013).

43. Global Alliance for Chronic Diseases (GACD) http://www.gacd.org/ projects/current-projects (accessed 14 Dec 2013). 